\title{
Autoestima y síndrome de burnout en médicos de unidades críticas. Estudio correlacional. Hospital Nacional Dos de Mayo. Lima-Perú
}

Contreras-Camarena Carlos* 1,2,a ; Lira-Veliz Humberto ${ }^{1,3, b}$; Benito-Condor Briam 4,c

RESUMEN

Objetivo: Determinar el rol de autoestima frente al síndrome de burnout (SBO) en médicos del Departamento de Emergencia y Cuidados Críticos del Hospital Nacional Dos de Mayo (HNDM). Lima-Perú, 2020.

Materiales y métodos: Estudio analítico y correlacional. Se encuestaron 66 médicos. Las variables autoestima y burnout se midieron con los test de Rosenberg y el cuestionario de Maslach MBI-HSS, respectivamente. La correlación de Pearson se empleó para determinar la correlación de variables principales y Odds ratio para establecer la probabilidad de aparición del burnout de acuerdo con el nivel de autoestima. Un valor de $p<0,05$ fue considerado como estadísticamente significativo. Resultados: El 16,66 \% mostró cansancio emocional; el 28,78 \%, despersonalización; y 25,75\%, baja realización personal. Las mujeres tuvieron menor realización personal y los varones mayor despersonalización. El 25,75 \% tuvieron autoestima baja y en este grupo la frecuencia de burnout fue tres a seis veces mayor. Se encontró una correlación negativa entre autoestima y cansancio emocional ( $r$ Pearson: - 0,551), correlación negativa entre autoestima y despersonalización ( $r$ Pearson: - 0,298) y, por el contrario, se encontró correlación positiva entre autoestima y realización personal ( $r$ Pearson: 0,499).

Conclusiones: Uno de cada cuatro médicos tuvo autoestima baja. El síndrome de burnout fue más frecuente en este grupo, comparado con el grupo de autoestima media-alta. El 71,21\% de los médicos tuvieron al menos una dimensión comprometida; $19,70 \%$, dos dimensiones, y 13,64 \% tuvieron SBO establecido. La autoestima baja favorecería el burnout, mientras que la autoestima alta tendría un rol protector.

Palabras clave: Autoestima; Burnout; Estrés laboral (Fuente: DeCS BIREME).

\section{Self-esteem and burnout syndrome among physicians of critical care units. A correlational study. Hospital Nacional Dos de Mayo. Lima-Peru.}

\section{ABSTRACT}

Objective: To determine the role of self-esteem against burnout syndrome (BOS) among physicians of the Department of Emergency and Critical Care of the Hospital Nacional Dos de Mayo (HNDM). Lima-Peru, 2020.

Materials and methods: An analytical and correlational study. Sixty-six (66) physicians were surveyed. The variables "selfesteem" and "burnout" were measured with the Rosenberg Self-Esteem Scale and the Maslach Burnout Inventory-Human Services Survey (MBI-HSS), respectively. The odds ratio was used to establish the probability of burnout according to the self-esteem level, and the Pearson correlation coefficient was used to determine the correlation of major variables. A value of $p<0.05$ was considered as statistically significant.

Results: Sixteen point six six percent (16.66\%) of the study population showed emotional fatigue; $28.78 \%$, depersonalization; and $25.75 \%$, low personal fulfillment. Females achieved lower levels of personal fulfillment and males, higher levels of depersonalization. Twenty-five point seven five percent $(25.75 \%)$ had low self-esteem and, in this group, the frequency of burnout was three to six times higher. A negative correlation was found between self-esteem and emotional tiredness (Pearson's r: -0.551), and between self-esteem and depersonalization (Pearson's r: -0.298). Conversely, a positive correlation was found between self-esteem and personal fulfillment (Pearson's r: 0.499).

Conclusions: One out of four physicians had low self-esteem. Burnout was more common in this group, compared to the medium-high self-esteem group. Seventy-one point two one percent $(71.21 \%)$ of the physicians had at least one compromised dimension, $19.70 \%$ had two compromised dimensions and $13.64 \%$ had an established BOS. Low self-esteem may favor burnout, while high self-esteem may play a protective role.

Keywords: Self concept; Burnout, psychological; Occupational stress (Source: MeSH NLM).

1 Hospital Nacional Dos de Mayo (HNDM), Departamento de Emergencia y Cuidados Críticos. Lima, Perú.

2 Universidad Nacional Mayor de San Marcos, Departamento Académico de Medicina. Lima, Perú.

3 Universidad de San Martin de Porres, Facultad de Medicina Humana. Lima, Perú.

4 Hospital Regional Felipe Arriola Iglesias, Servicio de Emergencia. Iquitos, Perú.

a Médico Internista, Doctor en Medicina.

b Médico Intensivista.

c Médico Cirujano.

*Autor corresponsal.

Horiz Med (Lima) 2021; 21(2): e1507 


\section{INTRODUCCIÓN}

En 1974, el psiquiatra germano-estadounidense Herbert J. Freudenberger describió por primera vez el síndrome de burnout (SBO) y lo definió como un complejo sintomático de naturaleza psicosocial que se presentaba en los trabajadores de salud debido a una demanda excesiva de energía (1). Más tarde, Maslach y Jackson elaboraron el cuestionario Maslach Burnout Inventory-Human Service Survey (MBI-HSS), que desde 1986 es el instrumento más por usado para cuantificar el SBO ${ }^{(2)}$. El burnout se presenta en trabajadores que tienen contacto frecuente y prolongado con personas. Las condiciones estresantes que promueven su aparición son el incremento en la demanda, la falta de recursos y las deficientes condiciones laborales. Dentro del personal de salud, los médicos constituyen un grupo vulnerable, sobre todo los que laboran en áreas críticas, por la gran exigencia asistencial y alto nivel de responsabilidad que demanda su trabajo ${ }^{(3)}$. El MBI-HSS realiza una evaluación tridimensional con ítems claramente establecidos por sus autoras: el cansancio emocional (agotamiento físico y psíquico), la despersonalización (actitud indiferente con los pacientes y sentimientos de cinismo) y la realización personal (autoevaluación negativa respecto a su propia habilidad y eficacia para el trabajo), cada uno con una sintomatología diferente. La prevalencia mundial del SBO fluctúa entre 40 a $60 \%$. Estados Unidos de Norteamérica reporta una prevalencia de alrededor del $50 \%(4,5)$. En la investigación realizada por Shanafelt T et al., el $54 \%$ de los médicos reportaron al menos un síntoma de burnout, casi el doble de la tasa de la población general estadounidense ${ }^{(5)}$.

En España, la prevalencia del SBO fluctúa entre 11,6 y $14,6 \%{ }^{(6)}$, en México es de 41,6 a $44 \%{ }^{(7)}$ y en Chile está entre $7,6 \%$ a $50 \%{ }^{(8)}$. En el Perú, la prevalencia es variable: de $3,7 \%{ }^{(9)}$ a $6,9 \%{ }^{(10)}$ hasta $80 \%$, como ocurre en un hospital de la amazonia ${ }^{(11)}$. Los estudios realizados en trabajadores de salud revelaron frecuencia incrementada del SBO en médicos de los servicios de emergencia y cuidados intensivos (12). En estos grupos, su presencia conlleva a disminución de la calidad de atención, descenso de productividad e incremento de errores médicos (OR: 2,72 IC 95\%: 2,19-3,37), comparados con médicos que no padecen el burnout (13). La exigencia cualicuantitativa de gerentes y de usuarios, el incremento de la demanda hospitalaria y la escasez de recursos facilitan la aparición de trastornos emocionales que favorecen los cuadros de SBO.

El enfoque transaccional como causa del burnout está relacionado con el estudio de variables intrapersonales, de conducta, responsabilidad y optimismo. La autoestima, el aceptarse como uno es, está relacionada a la maduración y el desarrollo psicológico. Dentro de este contexto, postulamos que un nivel adecuado de autoestima tiene un efecto favorable y también sería un modulador en la prevención y tratamiento del SBO ${ }^{(14)}$. Esta hipótesis nos llevó a realizar la presente investigacion, cuyo objetivo fue determinar el rol de la autoestima frente al síndrome de burnout (SBO) en médicos del Departamento de Emergencia y Cuidados Críticos del Hospital Nacional Dos de Mayo de Lima, Perú.

\section{MATERIALES Y MÉTODOS}

\section{Diseño y población de estudio}

Se realizó un estudio analítico correlacional con la finalidad de determinar la relación de autoestima con el síndrome de burnout. La población estuvo constituida por médicos pertenecientes al Departamento de Emergencia y Cuidados Críticos del Hospital Nacional Dos de Mayo. Participaron 25 intensivistas, 20 emergenciólogos y 21 internistas; todos ellos realizaban guardias hospitalarias en los servicios de Emergencia y Cuidados Intensivos. Los médicos que estaban de vacaciones, con licencias y ausentes por diferentes motivos al momento de realizar la encuesta fueron excluidos.

\section{Variables y mediciones}

Los datos se recolectaron a través de un cuestionario en el mes de marzo del 2020. Las variables sociodemográficas consideradas fueron edad, sexo, procedencia (Costa, Sierra, Selva), estado civil, etc. Además, se incluyeron otras variables potencialmente relacionadas con el síndrome de burnout como número de horas de trabajo por turno, número de hijos, docencia universitaria y atención en consultorio particular. Finalmente, se evaluó la presencia de idea suicida, que es un síntoma ominoso dentro del síndrome de burnout.

Para cuantificar el SBO se utilizó el cuestionario de Maslach MBI-HSS, de gran aceptación mundial, con estructuración factorial, validez interna e importante fiabilidad $(90 \%)^{(15)}$, modificado y validado para los profesionales de la salud en $1986^{(16)}$. Este instrumento evalúa tres dimensiones del burnout: el agotamiento emocional, con 9 preguntas (valor máximo: 54); la despersonalización, con 5 preguntas (valor máximo de 30), y la realización personal, con 8 preguntas (puntaje máximo 48). Se consideró presencia de SBO con puntuación $>26$ en la dimensión de cansancio emocional, $>9$ en despersonalización y < 34 en realización personal. El cansancio emocional se clasificó en bajo (8-18), medio (19-26) y alto (27-54); las categorías de la despersonalización fueron baja (0-5), media (6-9) y alta (10-30); finalmente, la realización personal se calificó como baja (0-33), media (34-39) y alta (40-48). Se determinó que los médicos mostraban un indicio de burnout cuando al menos una dimensión estaba alterada; si eran dos las dimensiones modificadas, entonces se trataba de una tendencia a burnout; por último, cuando las tres dimensiones estaban alteradas, estábamos frente a un SBO definido o establecido. En forma 
global, la presencia de SBO fue evidente en los participantes con una puntuación alta en los rubros de agotamiento emocional y despersonalización, y con puntaje bajo en realización personal. La autoestima se determinó con la escala de Rossemberg ${ }^{(17)}$, un instrumento unidimensional muy utilizado, y ya validado en español, que tiene una consistencia interna de 0,76 a 0,87 y una confiabilidad que fluctúa entre 75 y $80 \%$. Consta de diez preguntas cuyas respuestas están estructuradas en cuatro escalas: 1 (muy en desacuerdo), 2 (en desacuerdo), 3 (de acuerdo) y 4 (muy de acuerdo). Se consideró como autoestima baja una puntuación de 0 a 25; autoestima media, de 26 a 29; y autoestima alta, de 30 a 40 . La variable autoestima se hizo dicotómica y categorizamos la autoestima como baja cuando la puntuación fue < 26 (percentil 30).

\section{Análisis estadístico}

Los datos se procesaron con el paquete estadístico SPSS versión 22 y las encuestas incompletas fueron excluidas. Se determinaron las frecuencias, frecuencia absolutas y relativas para las variables categóricas; y para las variables numéricas principales, la media, desviación estándar y los percentiles 30,60 y 90 . Las variables se dicotomizaron para construir tablas de $2 \times 2$. Empleamos Odds ratio como estimado del riesgo relativo e IC $95 \%$. La correlación entre la variable autoestima con cada una de las dimensiones del síndrome de burnout se determinó con el test de Pearson. Además, se realizó análisis de regresión lineal múltiple. El valor de $p<0,05$ fue considerado como significativo.

\section{Consideraciones éticas}

La investigación fue aprobada por el Comité de Ética del Hospital Nacional Dos de Mayo. Las encuestas se aplicaron con una autorización previa de los participantes. Asimismo, el anonimato de los encuestado se guardó en cada etapa del proceso.

\section{RESULTADOS}

Las características de la población se muestran en la tabla 1. La edad media fue $41,71 \pm 6,25$; la mediana, 41 ; y la moda, 39. El $87,88 \%$ de los participantes estudió el pregrado en una universidad pública. Sesenta y tres profesionales $(95,45 \%)$ tenían entre 1 a 2 hijos. El $54,55 \%$ eran originarios de la Costa; $42,42 \%$, de la Sierra; y 3,03\%, de la Selva. No se encontró asociación estadísticamente significativa entre las variables sociodemográficas y la presencia de SBO.

Respecto a la especialidad de los médicos, no hallamos diferencias significativas en las dimensiones de agotamiento emocional y realización personal $\left(\mathrm{X}^{2}: 4,35-\mathrm{p}: 0,11\right)$; sin embargo, en la segunda dimensión del burnout, los médicos de emergencia mostraron la mayor frecuencia. La despersonalización se observó en el 45,16 \% de emergenciólogos, el 35,48 \% de los intensivistas y el $19,35 \%$ de los internistas ( $\left.X^{2}: 7,20-p: 0,02\right)$.

Tabla 1. Características sociodemográficas y laborales. Departamento de Emergencia y Cuidados Críticos. HNDM. Lima-Perú (n: 66)

\begin{tabular}{|c|c|c|c|}
\hline \multicolumn{2}{|l|}{ Variables } & \multirow[b]{2}{*}{31} & \multirow{2}{*}{$\begin{array}{c}\% \\
46,97\end{array}$} \\
\hline Edad & $\leq 40$ & & \\
\hline & $>40$ & 35 & 53,03 \\
\hline \multirow[t]{2}{*}{ Sexo } & Masculino & 47 & 71,21 \\
\hline & Femenino & 19 & 28,79 \\
\hline \multirow[t]{2}{*}{ Estado civil } & Soltero & 34 & 51,52 \\
\hline & Casado & 32 & 48,48 \\
\hline \multirow[t]{2}{*}{ Duración de guardia hospitalaria } & 12 horas & 37 & 56,06 \\
\hline & 24 horas & 29 & 43,94 \\
\hline \multirow[t]{2}{*}{ Docencia } & Sí & 39 & 59,09 \\
\hline & No & 27 & 40,91 \\
\hline \multirow[t]{2}{*}{ Consultorio particular } & Sí & 10 & 15,15 \\
\hline & No & 56 & 84,85 \\
\hline \multirow[t]{2}{*}{ Universidad de origen } & Pública & 58 & 87,88 \\
\hline & Privada & 8 & 12,12 \\
\hline \multirow[t]{2}{*}{ N. ${ }^{\circ}$ de hijos } & $\leq 2$ & 63 & 95,45 \\
\hline & $\geq 3$ & 3 & 4,55 \\
\hline \multirow[t]{2}{*}{ Idea suicida } & Sí & 0 & 0,00 \\
\hline & No & 66 & 100,00 \\
\hline
\end{tabular}




\begin{tabular}{|c|c|c|c|}
\hline Variables & & $\mathbf{N}$ & $\%$ \\
\hline \multirow[t]{3}{*}{ Lugar de nacimiento } & Costa & 36 & 54,55 \\
\hline & Sierra & 28 & 42,42 \\
\hline & Selva & 2 & 3,03 \\
\hline \multirow[t]{3}{*}{ Especialidad } & Médico de emergencia & 20 & 30,30 \\
\hline & Intensivista & 25 & 37,88 \\
\hline & Internista & 21 & 31,82 \\
\hline
\end{tabular}

Once médicos $(16,66 \%)$ de la población estudiada tuvieron agotamiento emocional; diecinueve $(28,78 \%)$, despersonalización, y diecisiete $(25,75 \%)$, baja realización personal. Diecisiete médicos mostraron autoestima baja; este grupo, que en su mayoría estuvo conformado por mujeres, tuvo 3,5 veces más probabilidad de presentar cansancio emocional, alrededor de 6 veces más probabilidad de padecer de despersonalización y 4 veces más riesgo de presentar baja realización personal, comparados con el grupo de autoestima media-alta (Tabla 2).

Tabla 2. Autoestima y síndrome de burnout en médicos de unidades críticas. Hospital Nacional Dos de Mayo.Lima-Perú (n:66)

\begin{tabular}{|lrrrrrr|} 
& \multicolumn{1}{c}{ Cansancio emocional } & Despersonalización & \multicolumn{2}{c|}{ Realización personal } \\
\hline Autoestima & Burnout & Normal & Burnout & Normal & Burnout & Normal \\
\hline Baja & $\mathrm{n}(\%)$ & $\mathrm{n}(\%)$ & $\mathrm{n}(\%)$ & $\mathrm{n}(\%)$ & $\mathrm{n}(\%)$ & $\mathrm{n}(\%)$ \\
\hline Media-alta & $7(63,6)$ & $10(18,2)$ & $12(63,15)$ & $5(10,63)$ & $10(58,82)$ & $7(14,28)$ \\
\hline Riesgo relativo & $4(36,4)$ & $45(81,8)$ & $7(36,85)$ & $42(89,37)$ & $7(41,18)$ & $42(85,72)$ \\
\hline IC 95 \% & 3,50 & & 5,93 & & 4,11 & \\
\hline Total & $1,70-7,16$ & & $2,42-14,55$ & & $1,86-0,09$ & \\
\hline
\end{tabular}

Diecinueve médicos mostraron una puntuación que no calificaba para SBO. El 37,88 \% tuvieron indicio de burnout (una dimensión comprometida), el 19,70\% tuvieron tendencia al SBO (dos dimensiones comprometidas) y el 13,64 \% tuvieron el burnout establecido; es decir, encontramos alteración de las tres dimensiones: cansancio emocional, despersonalización y baja realización personal (Figura 1).

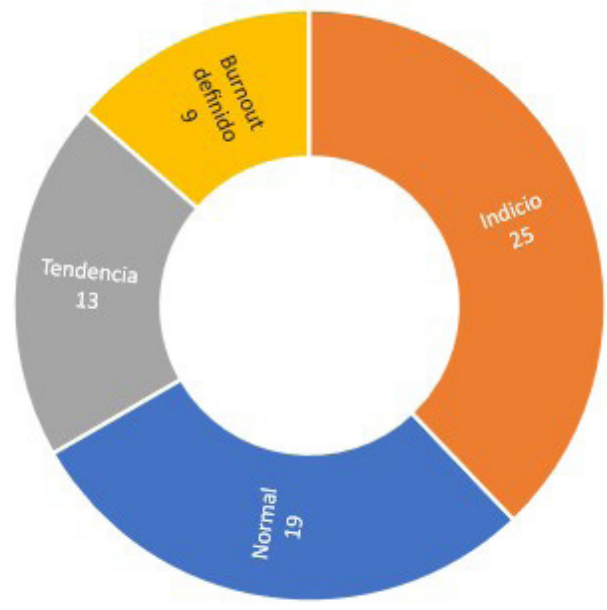

Figura 1. Síndrome de burnout en médicos del Departamento de Emergencia y Cuidados Críticos. HNDM. Lima-Perú (n: 66) 
La puntuación promedio encontrada para la autoestima fue de $27,36 \pm 6,33$, es decir, de nivel medio. Los percentiles 30,60 y 90 fueron 26,30 y 35 , respectivamente, las puntuaciones debajo del percentil 30 fueron considerados como críticas. El 25,75\% (17/66) mostró autoestima baja; y el 74,25\%, media-alta. El 47,36\% (9/19) de las mujeres tuvo autoestima baja, comparado con el 17,02 \% (8/47) de los hombres $(p: 0,01)$. La proporción de autoestima baja en menores de 40 años (14/31) fue mayor que en las personas de más de 40 años (3/35), este hallazgo fue estadísticamente significativo y muestra que los médicos mayores de 40 años tuvieron mayores niveles de autoestima. En el análisis bivariado, se encontró correlación positiva, es decir, a mayor edad, mayor autoestima ( $r$ de Pearson: 0,275; $p$ : 0,02). En el grupo de autoestima baja predominaron los solteros $(70,60 \%)$, mientras que en el grupo de autoestima media-alta la mayor proporción estuvo conformado por casados $(59,20 \%)$. No se encontraron diferencias significativas respecto a autoestima en las variables lugar de origen, universidad de procedencia, número de hijos y especialidad médica $(p>0,05)$.

El análisis correlacional entre autoestima y las dimensiones del burnout determinó una correlación negativa entre autoestima y agotamiento emocional ( $r$ de Pearson: - 0,511) y una correlación negativa entre autoestima y despersonalización ( $r$ de Pearson: - 0,298), por lo que se asume que, a menor autoestima, existe mayor nivel de agotamiento y despersonalización. Por el contrario, la relación encontrada entre autoestima y realización personal fue positiva ( $r$ de Pearson: 0,499), siendo hallazgos estadísticamente significativos $(p<0,05)$. Adicionalmente, mediante la regresión lineal simple se establecieron los coeficientes de determinación para evaluar la probabilidad predictiva de aparición del SBO: se encontró que la autoestima puede determinar y explicar la presencia de agotamiento emocional hasta en un $26,00 \%$; en el 0,08\%, de despersonalización; y el $0,02 \%$, de realización personal (Tabla 3).

Tabla 3. Correlación entre autoestima y síndrome de burnout en médicos de unidades críticas. Hospital Nacional Dos de Mayo. Lima-Perú

\begin{tabular}{|c|c|c|c|c|}
\hline & & Cansancio emocional & Despersonalización & Realización personal \\
\hline Correlación de Pearson valor & & $-0,551$ & $-0,298$ & 0,499 \\
\hline de $p$ (bilateral) & & 0,00 & 0,01 & 0,00 \\
\hline Regresión lineal simple valor & $R^{2}$ & 0,261 & 0,089 & 0,024 \\
\hline de $p$ (bilateral) & $p$ & 0,00 & 0,00 & 0,00 \\
\hline
\end{tabular}

\section{DISCUSIÓN}

La prevalencia del SBO está "in crescendo" en el personal de salud, sobre todo en los médicos que laboran en unidades de emergencia. Debido a su grave repercusión personal, la organización Mundial de la Salud (OMS), recientemente, ha propuesto la inclusión del SBO como una entidad nosológica (QD85) en la clasificación de enfermedades internacional (CIE-11), que entraría en vigor el $1 .^{\circ}$ de enero del $2022^{(18)}$. La elevada prevalencia del burnout no solo provoca disfunción personal, sino que ocasiona graves consecuencias laborales y económicas (genera costos indirectos al sistema de salud), y promueve el ausentismo laboral.

No se encontraron diferencias significativas entre las variables sociodemográficas y laborales en relación a la aparición del SBO. Estos hallazgos podrían sugerir que los factores extrínsecos organizacionales tendrían mayor relevancia que los factores personales en la génesis del síndrome, desplazando las cualidades personales a un segundo plano, lo que sería importante para el enfoque terapéutico que estaría dirigido, principalmente, a las deficiencias institucionales más que a los trastornos personales.
La ausencia de asociación entre variables sociodemográficas y burnout difiere de lo reportado por otros autores que sí encontraron algún grado de asociación entre edad, sexo, raza y procedencia urbana rural con el SBO $(6,12,19)$. Estas diferencias, probablemente, están relacionadas al tamaño y heterogeneidad de la población, predominancia de algún género y categorización del síndrome. Así, para Manzano y Ramos (19) existe una asociación entre menor edad y SBO, cuya elevada frecuencia en este grupo etario sería consecuencia de la escasa experiencia, menor calificación profesional y mayor estrés. En la investigación de Merces et al. (20) el grupo más numeroso estaba conformado por mujeres (razón F/M: 6,43) y encontraron una correlación significativa entre sexo femenino y el SBO.

La falta de asociación entre las variables sociodemográficas y el SBO encontrada en nuestra investigación concuerda con los hallazgos de Vásquez Fonseca et al. (21), quienes, además, reportaron una importante correlación negativa entre SBO y satisfacción laboral, hallazgo que sugiere la existencia de otras variables psicoafectivas que influirían en la aparición del burnout. Gran parte de 
estudios publicados al respecto involucraron poblaciones heterogéneas del personal de salud. Creemos que otros factores conductuales positivos, como motivación, asertividad, afectividad y resiliencia, desempeñarían un importante rol en el afrontamiento del SBO. Debido a la disparidad encontrada en potenciales factores de riesgo para burnout, es necesario diseñar investigaciones con poblaciones numerosas, más homogéneas, para obtener resultados concluyentes.

En nuestro estudio, la prevalencia del burnout definido fue $13,64 \%$. Este hallazgo difiere de Aldave (Perú), que encontró un $27,80 \%$ de burnout definido ${ }^{(22)}$, y de Bresó (Colombia), que reportó $25,40 \%$ de casos de este cuadro ${ }^{(23)}$. En el primer caso, la diferencia sería consecuencia de la heterogeneidad de la población, ya que incluyeron diversas especialidades (57 \% de especialidades quirúrgicas), pues la frecuencia del SBO varía según la especialidad. En el segundo caso, la desigualdad podría explicarse por la menor edad de la población, pues los jóvenes estarían más relacionados con el burnout, probablemente, por menor autoestima y experiencia.

El mayor porcentaje de despersonalización lo encontramos en el grupo de los médicos de emergencia $(45,16 \%)$, valor que difiere de los resultados de Vílchez et al. (11), que reportaron una prevalencia extremadamente alta de despersonalización $(96,7 \%)$ en los médicos de Ucayali y una elevada proporción de realización personal baja (76,7 \%): estos resultados se explicarían porque el 33,3\% de la población del estudio estaba formada por médicos generales, quienes tendrían una mayor alteración de esta variante. La mayor proporción de despersonalización encontrada en emergenciólogos sería consecuencia de la falta de seguridad, alteración del clima laboral y violencia a la que están expuestos. Por la naturaleza de su trabajo, ellos constituyen la primera línea de atención y es frecuente que sean agredidos verbal o físicamente: esta violencia lleva a un conflicto laboral, estrés y desmotivación del personal de salud.

Miret et al. ${ }^{(24)}$ señalan que el $60 \%$ del personal de salud fue víctima de violencia al menos una vez; el $45 \%$ de estos casos ocurrió en un servicio de emergencia, y los médicos y enfermeras fueron los grupos más vulnerables. En el Hospital Nacional Dos de Mayo no existe un reporte oficial de las agresiones al personal de salud, pero en nuestra práctica en el Servicio de Emergencia, la agresión al personal de salud es constante y es probable que alcance al 90 o $100 \%$ de los trabajadores en este servicio. Según un estudio peruano ${ }^{(24)}$, en una población de 200 médicos, el $84 \%$ fue agredido: $65 \%$ no reportó el hecho y en solo el 1,80 \% se realizó una investigación que concluyó sin sanción alguna. Estos sucesos alteran sus expectativas personales con la consiguiente aparición de irritabilidad, cinismo y distanciamiento (despersonalización), lo que trastorna la relación médico-paciente. Los médicos del Departamento de Emergencia y Cuidados Críticos mostraron mayor despersonalización que cansancio emocional, y tres de cada cuatro mostraron una autoestima media-alta en este grupo. Encontramos un nivel bajo de cansancio emocional y menor proporción de baja realización personal. Este resultado sugiere que un nivel de autoestima medio-alto regularía la manera en que se afecta la dimensión de realización personal, tal como lo sugieren otros autores, y también actuaría como un protector disminuyendo el cansancio emocional.

Es necesario investigar para valorar el rol de autoestima en la alta despersonalización, que fue una constante en estudios peruanos. Nuestros resultados coinciden con otros investigadores ${ }^{(21,22,25)}$ en que la despersonalización es la dimensión más comprometida. Ciertos modelos teóricos aseguran que el cansancio emocional sería el gatillo que desencadena el SBO, seguido de despersonalización que, al final, afectaría la realización personal. Por otro lado, algunos autores postulan que la despersonalización es el primer y principal desencadenante del síndrome de burnout (26). En este sentido, resulta crucial diseñar investigaciones longitudinales que aclaren estas preguntas.

Es posible que el nivel de autoestima media-alta encontrado en el $74,25 \%$ de los médicos en nuestro estudio se relacione a una población masculina predominante y de mayor edad, ambas variables asociadas, con frecuencia, a mayor autoestima ${ }^{(27)}$. Los médicos de este grupo tuvieron bajos niveles de cansancio emocional y despersonalización y, además, menor proporción en la dimensión de baja realización personal, comparados con el grupo de autoestima baja. Estos resultados fueron estadísticamente significativos $(p<0,05)$, lo que sugeriría un rol protector de la autoestima. Los valores de $r$ de Pearson comprobaron la correlación negativa entre la autoestima y el cansancio emocional y la despersonalización; por otro lado, hallamos una correlación positiva significativa con la realización personal. Las personas con mayor autoestima tendrían reacciones más tolerantes y adaptativas frente al estrés laboral, a diferencia de aquellos con baja autoestima, quienes tendrían estrategias deficientes para afrontar el estrés crónico. Más aún, creemos que, debido a la naturaleza poligénica de este síndrome, la baja autoestima, por sí sola, no explicaría la totalidad de los indicios y tendencias del SBO. Algunos estudios han encontrada relación entre obesidad y síndrome metabólico con el síndrome de burnout ${ }^{(28)}$. Asimismo, deben estudiarse otras variables psicométricas individuales como optimismo, personalidad, afectividad e inteligencia emocional que, al igual que la autoestima, podrían modular el SBO y, al mismo tiempo, actuar como variables confusoras (29). La prevención y erradicación del SBO deberían ser un objetivo de los servicios administradores de salud, ya que este cuadro no solo perjudica la salud del trabajador, sino también la de la empresa y la seguridad del paciente. El 
meta-análisis de García et al. ${ }^{(30)}$ reportó la relación inversa entre el burnout y la seguridad del paciente, los niveles altos del SBO trastornan la seguridad del paciente con una probabilidad superior al 66,4\%. En esta línea, las empresas deberían preocuparse del bienestar y satisfacción laboral de sus trabajadores. Un médico insatisfecho y con burnout tiene entre 2 a 8 veces más probabilidades de cometer errores en su práctica profesional.

Nuestro hallazgo de $0,00 \%$ de idea suicida podría explicarse por el alto nivel de autoestima de la población y una edad relativamente joven. La idea suicida es un síntoma ominoso que suele presentarse en las etapas avanzadas del SBO. Estados Unidos de Norteamérica tiene la mayor prevalencia de suicidios del personal de salud, según un meta-análisis de más de 6695 médicos que encontró $6,5 \%$ de idea suicida, característica muy temida y de pronóstico ominoso.

Debido a que el $71,21 \%$ tuvieron al menos una dimensión del burnout comprometida, es necesario plantear mecanismos de corrección de la gestión de recursos humanos, como la promoción del autodiagnóstico del SBO, el ejercicio físico, el coaching, entrenamiento en atención plena (mindfulness) y ayuda psicoterapéutica. Al inicio, las estrategias de intervención deberían estar centradas en el hospital y no en el trabajador, como es usual. El hospital debería evaluar periódicamente el bienestar de sus trabajadores, como sugieren Shanafelt y Noseworthy ${ }^{(31)}$, para mejorar las políticas de promoción, bonificación y equidad laboral, conseguir una adecuada calidad de vida del trabajador y alcanzar la humanización del trabajo. Debido a los niveles epidémicos del SBO, se desarrollaron diversas estrategias de intervención organizacional a nivel mundial con la finalidad de reducir la prevalencia del SBO. West C et al. ${ }^{(32)}$ realizaron una revisión sistemática y meta-análisis con 2944 médicos, 15 ensayos aleatorios y 37 estudios de cohorte, con búsquedas en las principales bases de datos (MEDLINE, Embase, PsycINFO, Scopus y Web of Science), para evaluar el impacto de las medidas correctivas institucionales. Los autores concluyeron que existe una disminución cuantitativa de la puntuación promedio del cansancio emocional de 23,82 a 21,17 (IC 95\%: 1,67-3,64) y de la despersonalización, de 9,05 a 8,41 (IC 95\%: 0,15-1,14). Ambos hallazgos fueron estadísticamente significativos $(p<0,05)$, lo que confirma que las medidas correctivas organizacionales son efectivas en reducir el SBO.

Concluimos que existe una relación directa entre autoestima y la realización personal, y una relación inversa con agotamiento emocional y despersonalización. El $25,75 \%$ de los médicos tuvieron una autoestima baja. La frecuencia del SBO en este grupo fue mayor, ya que mostró entre 3 a 6 veces más probabilidades de presentar cansancio emocional, despersonalización y baja realización personal, comparados con el grupo de autoestima media-alta. La baja autoestima favorecería la aparición del SBO y, por el contrario, el nivel elevado de autoestima jugaría un rol protector frente al síndrome de burnout. Debido al tamaño y la homogeneidad de la población, los resultados podrían ser extrapolables a médicos de otras unidades críticas de hospitales de nivel III de atención.

Contribuciones de los autores: Carlos Contreras Camarena participó en la elaboración de protocolo de investigacion, análisis y procesamiento de datos, discusión de resultados, búsqueda bibliográfica y redacción del informe. Humberto Lira Veliz y Briam Benito Condor participaron en la elaboración de fichas de entrevistas, recolección de datos, búsqueda bibliográfica y discusión de resultados.

Fuentes de financiamiento: El artículo ha sido financiado por los autores.

Conflicto de intereses: Los autores declaran no tener ningún conflicto de interés.

\section{REFERENCIAS BIBLIOGRÁFICAS}

1. Seidler A, Thinschmidt M, Deckert S, Then F, Hegewald J, Nieuwenhuijsen $\mathrm{K}$, et al. The role of psychosocial working conditions on Burnout and its core component emotional exhaustion - A systematic review. J Occup Med Toxicol. 2014; 9(1): 10-3.

2. Maslach C, Jackson SE. The measurement of experienced burnout. J Occup Behav. 1981; 2(2): 99-113.

3. Bragard I, Dupuis G, Fleet R. Quality of work life, burnout, and stress in emergency department physicians: a qualitative review. Eur J Emerg Med. 2015; 22(4): 227-34.

4. Yates SW. Physician Stress and Burnout. Am J Med. 2020; 133(2): 160-4.

5. Shanafelt TD, Hasan O, Dyrbye LN, Sinsky C, Satele D, Sloan J, et al. Changes in Burnout and Satisfaction with Work-Life Balance in Physicians and the General US Working Population Between 2011 and 2014. Mayo Clin Proc. 2015; 90(12): 1600-13.

6. Bouza E, Gil-Monte PR, Palomo E. Síndrome de quemarse por el trabajo burnout en los médicos de España. Rev Clin Esp. 2020; 220(6): 359-63.

7. Hernández García TJ. Burnout en médicos de un hospital del sector público en el Estado de Hidalgo. Agric Soc Desarro. 2018; 15(2): 161-72.

8. Bitran $M$, Zúñiga $D$, Pedrals N, Echeverría G, Vergara C, Rigotti A, et al. Burnout en la formación de profesionales de la salud en Chile: Factores de protección y riesgo, y propuestas de abordaje desde la perspectiva de los educadores. Rev Med Chile. 2019; 147(4): 510-7.

9. Marticorena-Quevedo J, Beas R, Anduaga-Beramendi A, MaytaTristán P. Prevalencia del síndrome de burnout en médicos y enfermeras del Perú, Ensusalud 2014. Rev Per Med Exp Salud Publica. 2016; 33(2): 241-7.

10. Muñoz-del-Carpio Toya A, Arias Gallegos WL, Caycho-Rodríguez T. Burnout syndrome in medicine men from Arequipa city (Perú). Rev Chil Neuro-Psiquiatr. 2019; 57(2): 139-48.

11. Vílchez-Cornejo, J, Romaní-Ojeda L, Ladera-Porta K, Marchand-Gonzales M. Síndrome de burnout en médicos de un hospital de la Amazonía Peruana. Rev Fac Med Hum. 2019; 19(4): 60-7.

12. García FE, Cova-Solar F, Bustos-Torres F, Reyes-Pérez É. Burnout y bienestar psicológico de funcionarios de unidades de cuidados intensivos. Duazary. 2018; 15(1): 23-38.

13. Olszewski R, Owoc J, Manczak M, Tombarkiewicz M. P4342 How 
burnout affects errors among physicians: meta-analysis. Eur Heart J. 2019; 40(Suppl. 1): 2578.

14. Hernandez L, Hernandez D, Barraye H. La autoestima como variable moduladora del desgaste profesional en especialistas en Medicina General Integral. Rev Cubana Med Gen Integr. 2014; 30(2): 149-59.

15. Maslach C, Jackson S. Maslach Burnout Inventory. 2nd edition. Consulting Psychologist Press: USA; 1986.

16. Gil-Monte P. Factorial validity of the Maslach Burnout Inventory (MBI-HSS) among Spanish professionals. Rev Saude Publica. 2005; 39(1): $1-8$.

17. W. W. Norton \& Company. Escala de autoestima de Rosenberg [Internet]. 2020. Disponible en: https://wwnorton.com/college/ psych/psychsci/media/rosenberg.htm

18. Organización Mundial de la Salud International Classification of Diseases, 11th Revision (ICD-11) [Internet]. OMS; 2020. Disponible en: http://www.who.int/classifications/icd/en/

19. Manzano G, Ramos F. Profesionales con alto riesgo de padecer Burnout: enfermeras y secretarias. Rev Psiquiatria. 2001.

20. Merces MC, Freitas Coelho JM, Lua I, Silva DS, Tosoli Gomes AM, Erdmann AL, et al. Prevalence and factors associated with burnout syndrome among primary health care nursing professionals: a crosssectional study. Int J Environ Res Public Health. 2020; 17(2): 474.

21. Vásquez Fonseca VM, Salgado A, Gómez Pasos JC, Martinez J. Burnout y satisfacción laboral en médicos y enfermeras de un hospital de alta complejidad. Salud(i)Ciencia. 2018; 23: 325-31.

22. Aldave-Salazar MR. Síndrome de burnout y factores de riesgo en médicos Hospital Nacional Guillermo Almenara Irigoyen 2015 [Tesis]. Lima: Universidad de San Martín de Porres. Facultad de medicina humana; 2016.

23. Bresó-Esteves E, Pedraza-Álvarez L, Pérez-Correa K. Síndrome de burnout y ansiedad en médicos de la ciudad de Santa Marta. Duazary. 2019; 16(2): 259-69.

24. Miret C, Martínez Larrea A. El profesional en urgencias y emergencias: agresividad y burnout. Anales Sis San Navarra. 2010; 33(Suppl. 1): 193-201.

25. Muñoz A, Valdivia-Fernández B, Velásquez-Lazo C, ValdiviaRodríguez M, Arroyo-Tovar G, Toia-Larsen M, et al. Violencia contra médicos en hospitales de Arequipa, Perú 2016. Acta Med Perú. 2016; 33(2): 99-104.

26. Leiter MP. Burnout as a Developmental Process: Consideration of models [Internet]. Routledge; 2017; pp. 237-50. Disponible en: https://www.taylorfrancis.com/

27. Bleidorn W, Arslan RC, Denissen JJ, Rentfrow PJ, Gebauer JE, Potter $J$, et al. Age and gender differences in self-esteem: a cross-cultural window. J Pers Soc Psychol. 2016; 111(3): 396-410.

28. Ortiz MS, Sapunar J, Ortiz MS, Sapunar J. Estrés psicológico y síndrome metabólico. Rev Médica Chile. 2018; 146(11): 1278-85.

29. Yebra Delgado S, García Faza V, Sánchez Calvo A, Suárez Gil P, González Gómez L. Relación entre la inteligencia emocional y el burnout en los médicos de Atención Primaria. Semergen. 2020; 46(7): 472-8.

30. Garcia CL, Abreu LC, Ramos JL, Castro CF, Smiderle FR, Santos JA, et al. Influence of Burnout on patient safety: systematic review and meta-analysis. Medicina (Mex). 2019; 55(9): 553.

31. Shanafelt TD, Noseworthy JH. Executive leadership and physician well-being: nine organizational strategies to promote engagement and reduce burnout. Mayo Clin Proc. 2017; 92(1): 129-46.

32. West CP, Dyrbye LN, Erwin PJ, Shanafelt TD. Interventions to prevent and reduce physician burnout: a systematic review and meta-analysis. Lancet. 2016; 388(10057): 2272-81.
Correspondencia:

Carlos W. Contreras Camarena.

Dirección: Av. Oscar R Benavides 3046. A-403. Lima 1, Perú. Teléfono: +51912543776

Correo electrónico: ccontrerasc4@unmsm.edu.pe

Recibido: 30 de noviembre de 2020

Evaluado: 22 de enero de 2021 Aprobado: 17 de febrero de 2021

(c) La revista. Publicado por Universidad de San Martín de Porres, Perú. (c) ${ }_{\text {BY }}$ Licencia de Creative Commons Artículo en acceso abierto bajo términos de Licencia Creative Commons Atribución 4.0 Internacional. (http://creativecommons.org/licenses/by/4.0/)

ORCID iDs

Contreras-Camarena Carlos Thttps://orcid.org/0000-0002-7394-995X

Lira-Veliz Humberto

(1) https: / / orcid.org/0000-0003-0634-9927

Benito-Condor Briam

(1) https://orcid.org/0000-0003-3163-237X 